\title{
THE FORMATION OF THE ECCENTRIC-ORBIT MILLISECOND PULSAR J1903+0327 AND THE ORIGIN OF SINGLE MILLISECOND PULSARS
}

\author{
S. Portegies Zwart ${ }^{1}$, E. P. J. van den Heuvel ${ }^{2}$, J. van Leeuwen ${ }^{3}$, and G. Nelemans ${ }^{4}$ \\ ${ }^{1}$ Leiden Observatory, Leiden University, P.O. Box 9513, 2300 RA Leiden, The Netherlands \\ 2 Astronomical Institute “Anton Pannekoek," Science Park 9041098 XH Amsterdam, The Netherlands \\ ${ }^{3}$ Stichting ASTRON, P.O. Box 2, 7990 AA Dwingeloo, The Netherlands \\ ${ }^{4}$ Department of Astrophysics, Radboud University Nijmegen, Heyendaalseweg 135, NL-6525 AJ Nijmegen, The Netherlands \\ Received 2010 December 28; accepted 2011 March 11; published 2011 May 25
}

\begin{abstract}
The millisecond pulsar (MSP) J1903+0327 is accompanied by an ordinary G dwarf star in an unusually wide ( $P_{\text {orb }} \simeq 95.2$ days $)$ and eccentric $(e \simeq 0.44)$ orbit. The standard model for producing MSPs fails to explain the orbital characteristics of this extraordinary binary, and alternative binary models are unable to explain the observables. We present a triple-star model for producing MSPs in relatively wide eccentric binaries with a normal (main-sequence) stellar companion. We start from a stable triple system consisting of a low-mass X-ray binary (LMXB) with an orbital period of at least 1 day, accompanied by a $\mathrm{G}$ dwarf in a wide and possibly eccentric orbit. Variations in the initial conditions naturally provide a satisfactory explanation for the unexplained triple component in the eclipsing soft X-ray transient $4 \mathrm{U} 2129+47$ or the cataclysmic variable EC 19314-5915. The best explanation for J1903+0327, however, results from the expansion of the orbit of the LMXB, driven by the mass transfer from the evolving donor star to its neutron star companion, which causes the triple eventually to become dynamically unstable. Using numerical computations we show that, depending on the precise system configuration at the moment the triple becomes dynamically unstable, the ejection of each of the three components is possible. If the donor star of the LMXB is ejected, a system resembling J1903+0327 will result. If the neutron star is ejected, a single MSP results. This model therefore also provides a straightforward mechanism for forming a single MSP in the Galactic disk. We conclude that the Galaxy contains some 30-300 binaries with characteristics similar to J1903+0327 and about an order of magnitude fewer single MSPs produced with the proposed triple scenario.
\end{abstract}

Key words: binaries: close - methods: numerical - stars: individual (J1903+0327) - stars: neutron

Online-only material: color figures

\section{INTRODUCTION}

The classic channel for the formation of a millisecond pulsar (MSP) requires a close binary with an extreme mass ratio $(\lesssim 2 / 10)$. This binary survives a common-envelope evolution and the subsequent supernova explosion of the primary star. The neutron star (NS), formed in the supernova, can subsequently be spun up to an MSP in a phase of mass transfer from the $\lesssim 2 M_{\odot}$ Roche lobe-filling companion star, which in the process is slowly stripped from its envelope. During this phase the binary is visible as a low-mass X-ray binary (LMXB), eventually resulting in an MSP that is accompanied by a low-mass white dwarf in a relatively wide, almost circular orbit $\left(e \lesssim 10^{-3}\right)$ (Bhattacharya \& van den Heuvel 1991). In the Galaxy 50 such systems are known, while about 15 MSPs in the Galaxy have no companion at all (Lorimer 2008).

The formation of the recently observed binary MSP J1903+0327 cannot be reconciled with the above scenario. Its characteristics are too different: the companion star is a $\mathrm{G}$ dwarf instead of a white dwarf, and the orbit is highly eccentric, $e \simeq 0.44$ instead of the expected $\lesssim 10^{-3}$ (Cordes \& Chernoff 1998). In addition, the average mass of the companion of known Galactic MSPs with pulse period $<10 \mathrm{~ms}$ is $0.22 \pm 0.17 M_{\odot}$, whereas for J1903+0327 the companion mass is $1.03 M_{\odot}$ (Freire et al. 2009); and binary MSPs with a companion mass $>0.6 M_{\odot}$ tend to have a long pulse period $\langle P\rangle=$ $62 \pm 76 \mathrm{~ms}$ and short orbital periods ( $P_{\text {orb }}=5.2 \pm 4.8$ days $)$, whereas J1903+0327 has an extremely short pulse period of $2.15 \mathrm{~ms}$ and an extraordinary long orbital period of 95 days. These discrepancies with respect to the expected outcome of the standard scenario for producing an MSP in a binary requires an exotic solution.
We propose that J1903+0327 was born as a rather ordinary triple star of which the inner binary is the progenitor of an LMXB, and with an outer (tertiary) star that initially is less massive than the secondary so that the inner secondary evolved first. After a common-envelope phase and a supernova explosion, mass transfer in the inner binary leads to expansion of its orbit. Depending on the orbit of the outer star a dynamical instability ensues in which one of the three stars is ejected. Such an evolution results in an MSP binary with an outer companion in either a wide orbit (if the instability is avoided), or an MSP binary if the outer companion is ejected, or a normal binary plus a single MSP if the NS is ejected, or if the inner secondary is ejected, an MSP with a low-mass companion in an eccentric orbit, like J1903+0327.

The scenario sounds exotic, and it is, but in Sections 3 and 5 we estimate the available parameter space and calculate that this model leads to an acceptable birthrate. (We note that in a recent paper, Freire et al. (2009) independently also suggest that this model is one of the possible triple-star models for J1903+0327.) We will discuss the details of our scenario in Section 2 and quantify the results by performing simulations of triple-star systems in Section 4. We discuss the shortcomings of earlier proposed scenarios for the formation of J1903+0327 in Section 6 and summarize our conclusions in Section 7.

\section{THE TRIPLE SCENARIO FOR FORMING J1903+0327}

We propose that the binary J1903+0327 was born as a rather ordinary triple star. We envision a configuration at birth where a relatively massive $9-12 M_{\odot}$ primary star was orbited by a secondary star of $\sim 0.8-2.0 M_{\odot}$ in a relatively close $\left(\sim 200 R_{\odot}\right)$ 
orbit, and a tertiary star which is less massive than the secondary and has a rather wide $\gtrsim 560 R_{\odot}$ orbit around the inner binary. Adopting a tertiary mass that exceeds the secondary would considerably change the outcome of the evolution, because in that case it is the outer star that ascends the giant branch and possibly fills its Roche lobe before the inner binary has turned into an LMXB. The evolution of such triples has not yet been subjected to any study (Eggleton \& Kiseleva 1996; Eggleton 2006).

When the massive primary of the initial triple ascends the red-giant branch a common-envelope ensues (Webbink 1984; Taam \& Sandquist 2000; Deloye \& Taam 2010; Ge et al. 2010), in which the inner secondary and the degenerate core of the giant spiral in toward each other. A few Myr later, the stellar core explodes as a supernova, forming an NS with a low-mass companion in an eccentric orbit with a period of a few to several tens of days (Deloye \& Taam 2010). The outer orbit is likely influenced both by the mass shell ejected in the common envelope and by the supernova explosion. The mass shell containing 6-10 $M_{\odot}$ may slow down the outer companion star, which may cause its orbit to shrink, but the removal of mass from the system may also widen it. The supernova explosion causes the inner binary to receive a velocity kick (Blaauw 1961; Dewey \& Cordes 1987), which changes the orbits and may disrupt the triple. The kick results from two subsequent effects; the mass loss in the explosion and the intrinsic velocity kick imparted to the newly formed NS (see Section 5.2 for details). In the case of an electron-capture supernova, the latter effect is expected to result in a relatively low-velocity kick $<50 \mathrm{~km} \mathrm{~s}^{-1}$ (Dewi et al. 2005), which generally suffices to keep the outer orbit bound by compensating the effect of the mass loss in the supernova. In the surviving systems mass transfer will ensue in the inner binary after several Gyr. This rapidly circularizes the inner orbit by tidal forces and turns the system in an LMXB resembling the bright Galactic bulge LMXBs (Webbink et al. 1983; Taam 1983). An example of such a triple is 4U 2129+47 (V1727 Cyg), in which a $5.24 \mathrm{hr}$ LMXB is accompanied by a spectral type $\mathrm{F}$ dwarf in an eccentric orbit of about 175 days (Garcia et al. 1989; Bothwell et al. 2008; Lin et al. 2009). For our model we require that, contrary to $4 \mathrm{U} 2129+47$, at the moment of RLOF the LMXB has a period of at least $\sim 1$ day and will evolve to longer periods, rather than shrink due to the emission of gravitational waves (Pylyser \& Savonije 1988, 1989). In the case of $4 \mathrm{U} 2129+47$, the inner orbit will not expand due to the mass transfer and therefore will not perturb the outer orbit.

Mass transfer in the inner binary causes the accreting NS to be spun up to a millisecond spin period (Alpar et al. 1982; Radhakrishnan \& Srinivasan 1982). After several tens of millions of years, the expansion of the inner orbit leads to a dynamically unstable situation with respect to the orbit of the outer star. Depending on the precise configuration at the moment when the system becomes dynamically unstable, either the donor of the LMXB, or the outer tertiary star or the NS can be ejected from the unstable triple. In the latter case a single MSP results, and in the first-mentioned case a wide eccentric millisecond binary pulsar with a $\mathrm{G}$ dwarf companion is produced, resembling $\mathrm{J} 1903+0327$.

\section{CONSTRAINING THE BIRTH CONDITIONS FOR J1903+0327}

We investigate the conditions under which the proposed triple scenario produces a system similar to J1903+0327. With the currently observed orbital parameters of J1903+0327 $(a \simeq$
$123.2 R_{\odot}, e \sim 0.44, m_{G} \simeq 1.03 M_{\odot}$, and $M_{\mathrm{MSP}} \simeq 1.67 M_{\odot}$; Freire et al. 2009), the binding energy of the binary is about $E_{b} \simeq 2.65 \times 10^{46} \mathrm{erg}$. This poses a minimum to the binding energy of the triple just before it became dynamically unstable, and allows us to calculate the orbital separation of both the inner and the outer orbits at the moment that the dynamical instability sets in. In practice the binding energy of the stable triple will be higher than $E_{b}$ by the ratio of the masses of the ejected star with respect to the triple, or $\sim 20 \%$ (Heggie 1975; Fregeau et al. 2004), because the escaping star carries off some fraction of the binding energy to infinity.

The requirement that the binding energy of the triple must exceed $E_{b}$ allows us to calculate the orbital separation of both the inner and the outer orbits at the moment that the dynamical instability sets in. The criterion for dynamical stability sensitively depends on the separation and the eccentricity of the inner and outer orbits and on the masses of the three stars (Mardling \& Aarseth 2001). However, for the observed system J1903+0327 only the masses of two of the stars (the MSP and its current G dwarf companion) are known, as the initial secondary star was ejected from the binary.

The initial donor in the LMXB must have been more massive than the current MSP companion in order to evolve first, but not so massive that mass transfer in the LMXB would be unstable; we therefore adopt a mass of the original close companion of the NS of 1.0-2.0 $M_{\odot}$ (In principle a secondary mass as low as $0.8 M_{\odot}$ would suffice to warrant the inner binary to evolve into an LMXB, but in that case the tertiary star should be $<0.8 M_{\odot}$, which is smaller than the observed $1.03 M_{\odot}$ ). For the NS, we adopt a mass of $1.30 M_{\odot}$ before it starts accreting mass (Schwab et al. 2010), and in the same range of masses, but less massive than the inner secondary star for the outer companion. In the specific case of $\mathrm{J} 1903+0327$, the observed star is an $1.03 M_{\odot}$ $\mathrm{G}$ dwarf, which in our scenario would be identical to the initial tertiary star. The best match to the orbital characteristics are then obtained when we adopt the initial secondary mass to be $\sim 1.1 M_{\odot}$.

During the LMXB phase, mass is transferred from the inner companion (donor) to the NS. The time-averaged mass-transfer rate can be estimated from the empirical relation based on the initial orbital period of the LMXB (Shore et al. 1994). In our model, we assume that the NS accretes at most at the Eddington limit; for which we adopted $\dot{m}=1.5 \times 10^{-8} M_{\odot} \mathrm{yr}^{-1}$. The leftover mass that is provided by the donor but that is not accreted by the NS is assumed to leave the inner binary with the specific orbital angular momentum of the NS (Portegies Zwart 1995). The currently observed mass of the NS is $1.67 M_{\odot}$, which indicates that in our model it must have accreted about $0.37 M_{\odot}$ and that the donor star must have lost at least that same amount of mass. Mass transfer in such a binary system causes the orbit to expand quite dramatically, in particular if the mass transfer was not conservative.

We can now determine the separation of the inner and the outer orbit for the initial triple (before the LMXB phase) by an iterative procedure under the constraints that the total binding energy of the triple at the moment it became dynamically unstable is known, and by taking the effect of the mass-transfer process during the LMXB phase into account. Here we assumed that the mass lost from the LMXB leaves the triple in the form of a stellar wind. The resulting orbital constraints of this iterative procedure are presented in Figure 1 as the solid and dashed curves; these give the most likely initial conditions for forming J1903+0327. 


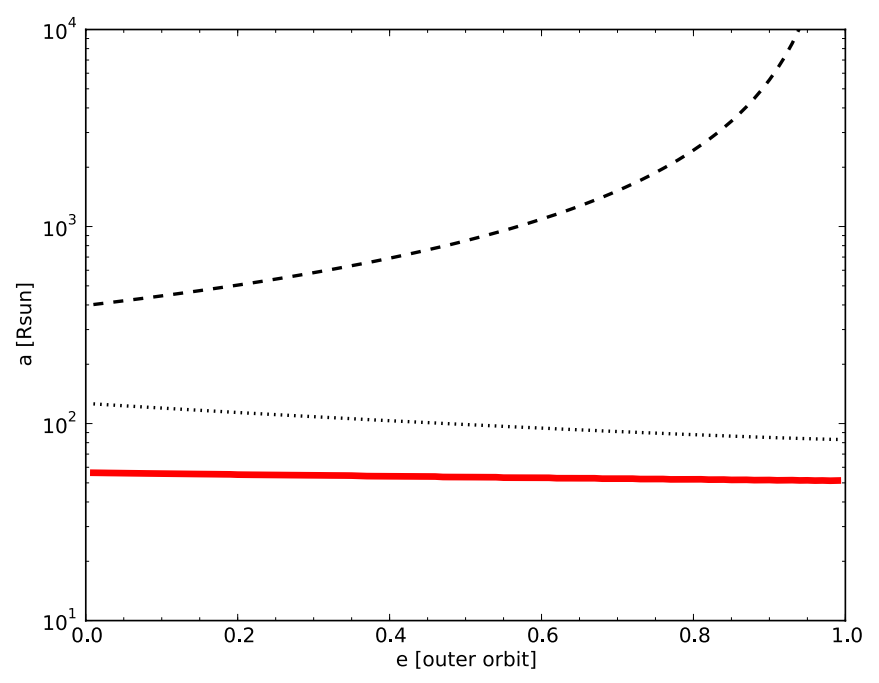

Figure 1. Triple with a $1.3 M_{\odot}$ neutron star and $1.1 M_{\odot} \mathrm{G}$ dwarf in a circular inner orbit (thick solid curve (red)) and a $1.0 M_{\odot} \mathrm{G}$ dwarf in an eccentric outer orbit (dashes) is stable and has the same binding energy as J1903+0327. These curves are calculated using the iterative procedure described in Section 3. The orbit of the LMXB expands as a consequence of the mass that is transferred from the initial secondary donor star to the neutron star. According to the analytic expression provided by Mardling \& Aarseth (2001), their Equation (90), the triple becomes dynamically unstable as soon as the orbital separation of the LMXB reaches the dotted curve. At this moment the triple dissolves and one of the three stars is ejected, leaving behind a binary with an orbital separation roughly somewhere between the dotted and the dashed (black) curve (see Figure 3).

(A color version of this figure is available in the online journal.)

With the semi-analytic procedure just described we still cannot predict the consequence of the dynamical instability, in particular because the final stage of the triple is highly dynamic and the orbital parameters do not allow us to predict the identity of the ejected star from first principles. However, one can imagine that it is quite likely that the less massive donor $\left(<0.73 M_{\odot}\right.$ when we adopt an initial secondary mass of $\left.1.1 M_{\odot}\right)$ is ejected, rather than the more massive MSP $\left(\sim 1.67 M_{\odot}\right)$ or the outer $\operatorname{star}\left(\sim 1.03 M_{\odot}\right)$. At the moment when the dynamical instability sets in the donor still has part of its hydrogen envelope, which explains why its mass exceeds that of the degenerate helium core.

\section{SIMULATING MASS TRANSFER IN A TRIPLE SYSTEM}

We quantify our proposed formation channel for J1903+0327 by performing extensive computer simulations of triple systems, starting at the onset of the LMXB phase. The calculations were performed using the Astrophysics Multipurpose Software Environment (AMUSE ${ }^{5}$; Portegies Zwart et al. 2009).

In the AMUSE environment we resolve the dynamical evolution of the triple star by a specialized numerical orbit integration, which is written in FORTRAN77. The mass transfer in the inner binary is implemented in Python. The outer star is treated as a point-mass and was not evolved during the simulation. The coupling between the numerical orbit integration of the three stars and the stellar evolution calculations is realized using AMUSE (Portegies Zwart et al. 2009). The most important role of AMUSE is converting the units and to realize the communication between the two codes. The former is done with a specialized unit conversion module and for the latter we

\footnotetext{
5 See http://www.amusecode.org.
}

spawned the different processes using the Message Passing Interface to transfer the required data in the proper units and in discrete instances between the two codes.

\subsection{Numerical Method}

The orbits of the triple stars were integrated using a regularized version of the Burlish-Stoer integrator (Aarseth \& Zare 1974a, 1974b), keeping the numerical error at machine precision, and allowing a maximum relative energy error of $\mathrm{O}\left(10^{-14}\right)$ per integration of the outer orbit.

During the dynamical evolution, we resolved the mass transfer and consequential change in orbital parameters of the LMXB that is orbited by the outer star.

The rate of mass transfer in the inner binary is calculated from the empirical relation based on the orbital period at the onset of RLOF in the LMXB: $\langle\dot{m}\rangle=6 \times$ $10^{-10}\left(P_{\text {orb }}\right.$ (initial) $/ 1$ day) $M_{\odot} \mathrm{yr}^{-1}$ (Shore et al. 1994).

The NS was allowed to accrete at most at the Eddington rate and any surplus mass is assumed to leave the binary with the angular momentum of the accreting NS, but lost adiabatically from the triple.

We perform the mass transfer in the inner binary every time the outer orbit has had 10 revolutions, after which the numerical orbit integration was updated using the newly calculated orbital parameters which resulted from carrying out the mass transfer. We varied the interval between which mass transfer in the inner binary was conducted between every 1-1000 outer orbits, but this choice did not significantly affect the results. (In Section 5.3, we present the results of a series of simulations where we decoupled the mass-transfer process from the gravitational evolution after the triple has become dynamically unstable.)

\subsection{Results of the Simulations}

We initialized $10^{3}$ binaries and calculated the evolution for each up to an age of at most $10 \mathrm{Gyr}$ or until the mass of the donor star drops to the mass of the degenerate helium core for a population-II star $\left(\lesssim 0.4 M_{\odot}\right.$; Tauris \& Savonije 1999).

Each triple was initialized by randomly selecting the eccentricity of the outer orbit from the thermal distribution and the initial separations of the inner and outer orbits for each tripleevolution calculation are selected using the iterative procedure described in Section 3 (see the thick solid and dashed curves in Figure 1). For each simulated binary, we randomly selected the inclination of the inner orbit with respect to the outer orbit, the longitude of the ascending node, the argument of periastron, and the phases of the two orbits. The preference in the orbital elements introduced by the supernova explosion may affect the rate of ejected MSPs relative to those that stay in a binary, but we ignore that complication. We found no significant correlations between the final outcome of the simulations and longitude of the ascending node, the argument of periastron, or the phases of the two orbits. The anisotropic velocity caused by the mass loss in the supernova of the inner binary is therefore not expected to have a significant effect on the surviveability of the triple. During the evolution of the triples, small eccentricities $(\lesssim 0.1)$ are commonly induced in the inner orbit, particularly when the triple approaches the regime where it becomes dynamically unstable.

We stop a simulation when the eccentricity of the inner (LMXB) orbit exceeds 0.3 for more than $10^{5}$ years. Such high eccentricities can be induced shortly by a strong interaction with the outer star, which typically results in the break-up of 


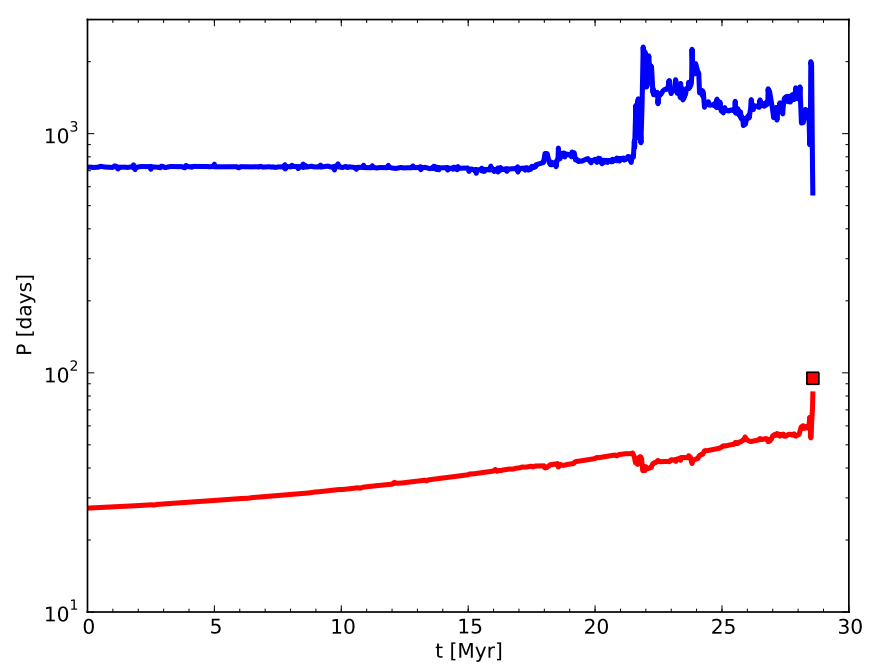

Figure 2. Evolution of the orbital period of a rather typical case which evolves to a system very similar to J1903+0327. The square (red) represents the final orbit of this simulation, which in this particular case resulted in a binary consisting of a $1.73 M_{\odot}$ MSP and a $1.0 M_{\odot}$ companion in a $\sim 100$ day orbit with $e \simeq 0.43$. The bottom line (red) indicates, as a function of time the evolution of the inner LMXB, whereas the top line (blue) represents the evolution of the outer orbit.

(A color version of this figure is available in the online journal.)

the triple, or by a long term secular perturbations of the inner orbit by the outer star (Kozai 1979). In the latter case, orbital variations result naturally from the secular evolution of the triple and do not directly lead to a dynamical instability. We still decided to terminate such simulations because it becomes hard to follow the mass-transfer process within the inner binary. It would require extensive hydrodynamical simulations to study the consequences of a Kozai resonances in a triple with a Roche lobe-filling inner binary. About $10 \%$ of our simulations were stopped as a consequence of this effect. Mass transfer in eccentric orbits is generally ill understood, although courageous attempts are underway to tackle this problem (Lajoie \& Sills 2011a, 2011b).

The majority (917) of the binaries become dynamically unstable long before the other stopping criteria apply, in which cases we continue to resolve the dynamics by integrating the equations of motion and resolving the internal mass transfer until one of the stars escapes. The orbital separation of the LMXB at which the triple is expected to become unstable is indicated by the dotted curve in Figure 1.

An illustrative example of the evolution of the period of the inner and outer orbits is presented in Figure 2. The evolution of the inner LMXB also drives the expansion of the outer orbit, in particular by the dynamical coupling between both orbits and in a lesser extend by the mass lost from the inner LMXB in those cases that mass transfer proceeds non-conservatively. This relative softening causes the final MSP binaries to be somewhat wider than observed in $\mathrm{J} 1903+0327$, and as a consequence the triple remains stable for somewhat longer than expected based on our analytic energy balance (see Section 3), and eventually results in the MSP being somewhat more massive (by about $0.2 M_{\odot}$ ) than observed in $\mathrm{J} 1903+0327$. We can compensate for this by adopting a slightly $(\sim 20 \%)$ smaller outer orbital separation at the onset of the LMXB phase.

The resulting parameters of the MSP with a G-star binary are presented in Figure 3; they straddle the observed orbital separation and eccentricity of J1903+0327. The phase of mass transfer for these binaries lasted for about 23.4 \pm 9.3 Myr, after

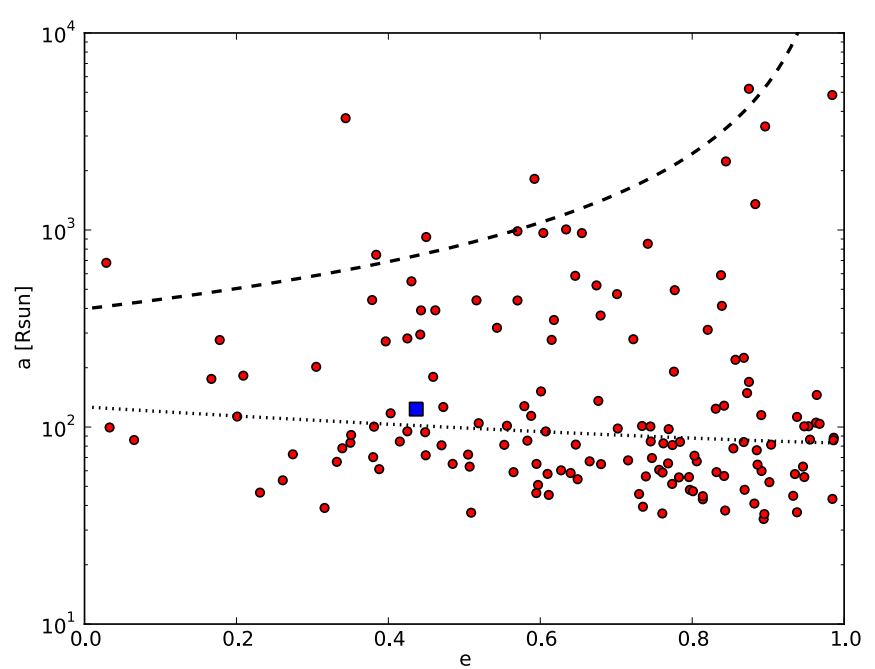

Figure 3. Eccentricity $(e)$ and semimajor axis $(a)$ of the MSP binaries with $\mathrm{G}$ dwarf companions that resulted our from simulations (dots (red), see Section 3). These binaries, initialized to mimic J1903+0327, are described in Section 3 and represented by the thick solid and dashed curves in Figure 1, which are reproduced here to guide the eye regarding the most likely range of final binary parameters. The square (blue) indicates the current orbital separation and eccentricity of J1903+0327. Note that the final eccentricity of the surviving binary is unrelated to the eccentricity of the initial outer orbit (black dashed curve).

(A color version of this figure is available in the online journal.)

which the donor was ejected in about one-fifth $\sim 20 \%$ of the cases. The resulting binaries, for those with $a<10^{4} R_{\odot}$, had an average orbital separation of $175 \pm 145 R_{\odot}$, and an eccentricity of $0.65 \pm 0.19$. During the LMXB phase, the NSs were able to grow from $1.30 M_{\odot}$ to $1.64 \pm 0.12 M_{\odot}$. The mean mass of the ejected donor was $0.72 \pm 0.13 M_{\odot}$.

Our success in reproducing the observed parameters of the binary MSP J1903+0327 demonstrates that its progenitor may well have been born as a triple star. However, the pin-pointed search of parameter space described here makes it impossible to derive the birthrate for $\mathrm{J} 1903+0327$, which we will calculate in the next section.

\section{HOW MANY J1903+0327-LIKE SYSTEMS ARE THERE IN THE GALAXY?}

We calculate the formation rate of J1903+0327-like systems by determining their birthrate with respect to that of ordinary LMXBs. The reason for this approach is the great uncertainty in the number of LMXB progenitors because binaries with such extreme mass ratios are observationally unknown.

This ratio depends on several factors.

1. The number of triple systems with suitable parameters.

2. The fraction of triples that ensues and survive the commonenvelope phase and the supernova explosion in which the NSs is formed.

3. The fraction of those triples that lead to J1903+0327-like systems (instead of single MSPs, classic MSP binaries or MSP binaries with a triple companion).

We will discuss each of these factors below in Sections 5.1, Section 5.2, and Section 5.3, respectively.

\subsection{The Number of Suitable Triple Stars}

According to the Hipparcos database, the ratio of hierarchical, higher order, multiple stellar systems (404) to binaries (1438) 


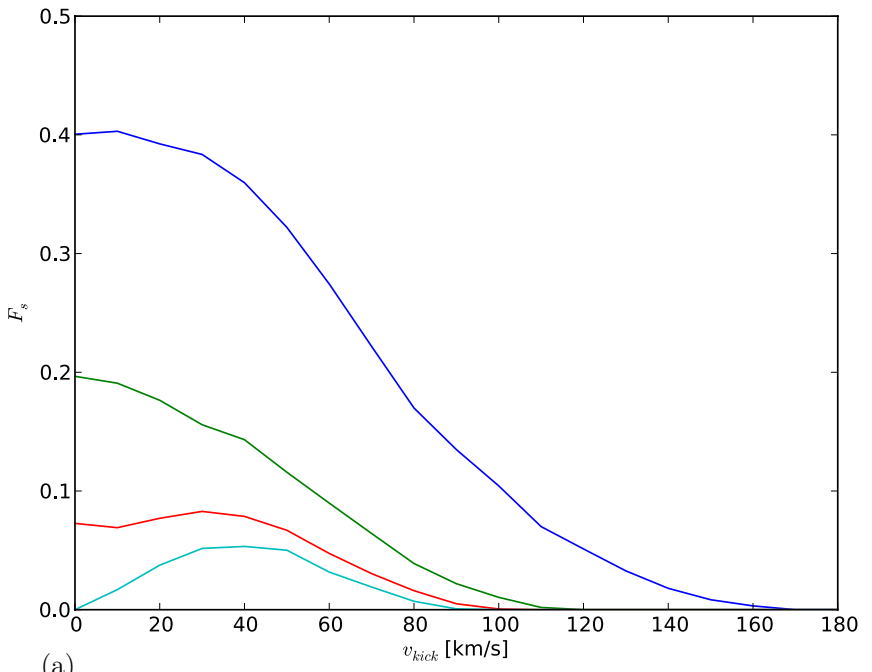

(a)

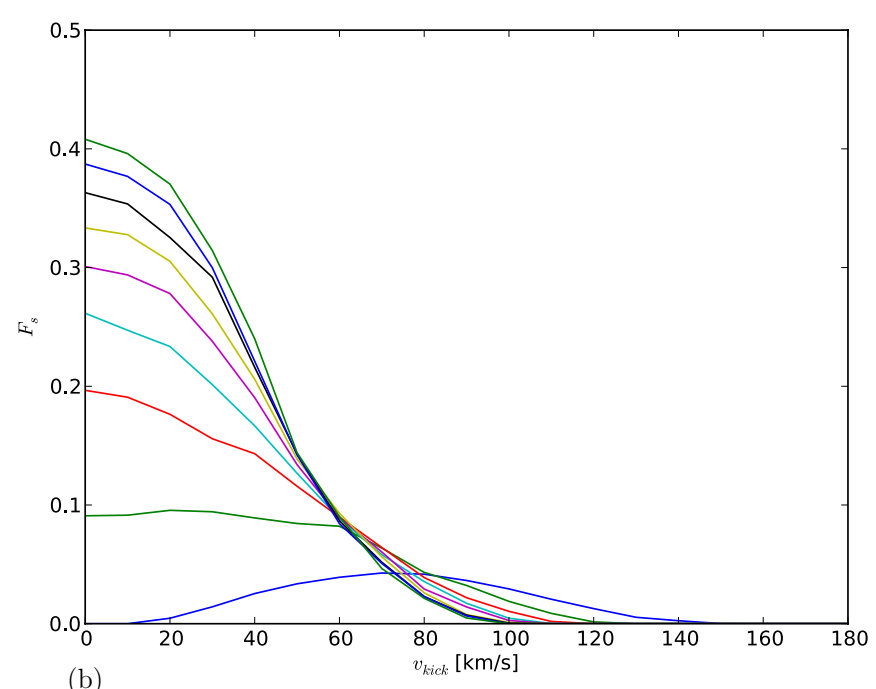

(b)

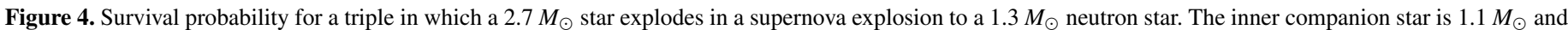

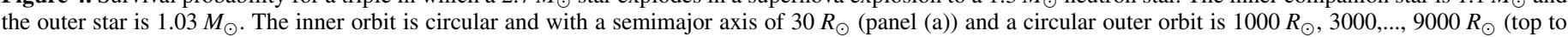

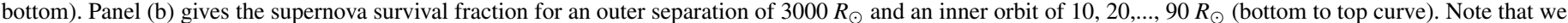
adopted here high mass loss in the supernova contrary to an electron-capture supernova, which suppresses the survival rate.

(A color version of this figure is available in the online journal.)

is $404 / 1842 \sim 0.22$ and the outer stars have a rather flat mass distribution (Eggleton \& Tokovinin 2008, 2010). We assume that the same ratio holds for (inner) binaries that are progenitors to LMXBs, even though there are none in this catalogue. We further require that the mass of the outer stars is about ten times lower than that of the inner binary. We then find a ratio of suitable triples to LMXB progenitor binaries of a few percent. We present in Section 6.2 observational evidence that such triples do exist.

\subsection{The Fraction of Triples That Survive the Supernova Explosion}

We will not dwell on the details of the common-envelope evolution, but assume that it leads to the spiral-in of the inner two components without significantly affecting the outer star. The consequences of the common envelope do not qualitatively affect our result, but have a strong effect on the derived birthrate (see Section 5.4). In Section 6.2 we discuss that the orbit of the tertiary star that orbits the LMXB 4U 2129+47 may have experienced a considerable reduction during the common envelope, but we consider this insufficient evidence to draw general conclusions regarding the effect of the common envelope on the outer orbit.

The effect of the supernova explosion in the inner binary on the orbital parameters of the triple can be calculated relatively straightforwardly, and has a profound effect on the survivability of the triple because the weakly bound outer orbit is easily disrupted.

The mass loss in the supernova explosion causes the inner binary to be ejected (Blaauw 1961; Boersma 1961). In order to keep the triple bound, a small asymmetric velocity kick imparted to the newly formed NS is required (Hills 1983; Brandt \& Podsiadlowski 1995; Tauris \& Takens 1998). In Figure 4, we show the survival probability of several combinations of inner and outer orbits as function of the asymmetric kick magnitude. In these calculations, the effect of the Blaauw-Boersma kick was self-consistently taken into account to calculate the survival probability. This fraction ranges from $\sim 40 \%$ for small kicks to zero for kicks significantly above $100 \mathrm{~km} \mathrm{~s}^{-1}$.
To study the future evolution of the triple through the LMXB phase, we need to derive the orbital parameters as a function of the most probable kick velocity and the amount of mass lost in the supernova. We consider several combinations of both, and the distinction between them can be made by the mass of the initial primary star and the moment it filled its Roche lobe.

In Section 3, we discussed the most likely range of parameters at birth and adopt these same numbers here to study the survivability of the triple. Here we make a distinction between two types of supernovae; in one case we adopt that the inner primary at the moment of the supernova is a $1.9 M_{\odot}$ helium star with a degenerate ONeMg core that is the leftover of the initial $9-12 M_{\odot}$ primary star. If the initial primary star was born somewhat more massive, $10-13 M_{\odot}$, and was stripped from its hydrogen envelope at a later stage of its evolution, its helium core may have grown to $\sim 2.7 M_{\odot}$. The ONeMg star that experiences an electron-capture supernova loses $\sim 0.6 M_{\odot}$ and if the exploding star has a more massive helium core, the mass loss is $\sim 1.4 M_{\odot}$. In both cases, we adopt an NS mass of $1.30 M_{\odot}$.

Apart from the smaller mass lost in the explosion of the ONeMg core, the NS is also expected to receive a smaller velocity kick upon birth. For these electron capture supernovae, we adopted a Gaussian distribution for the velocity kick with a dispersion of $20 \mathrm{~km} \mathrm{~s}^{-1}$ (Podsiadlowski et al. 2004; Scheck et al. 2004) in a random direction. From studies of single radio pulsars in the solar neighborhood, several kick velocity distributions have been constructed (Lyne \& Lorimer 1994; Hansen \& Phinney 1997; Cordes \& Chernoff 1998; Arzoumanian et al. 2002), most of which yield considerably higher velocity than for the ONeMg supernovae. These latter kick velocity distributions are expected to be more suitable for NSs formed from an isolated star or a non-interacting binary, in which case the NS is formed by the collapse of an iron core (van den Heuvel 2004; Podsiadlowski et al. 2004). These kicks range from single (zero-centered) Gaussian distributions with a velocity dispersion of $265 \mathrm{~km} \mathrm{~s}^{-1}$ (Hobbs et al. 2005) to more complicated distributions like the proposed two Gaussians with dispersions 
of $175 \mathrm{~km} \mathrm{~s}^{-1}$ and $700 \mathrm{~km} \mathrm{~s}^{-1}$ and relative probability of 0.86 and 0.14, respectively (Cordes \& Chernoff 1998).

We study the probability that a triple survives the supernova by means of Monte Carlo simulations. The simulated triples had the following characteristics: the inner binary consists of a $1.9 M_{\odot}$ helium star with a degenerate $\mathrm{ONeMg}$ core or a $\sim 2.7 M_{\odot}$ helium star, and a $0.8-2.0 M_{\odot}$ secondary. The latter was selected randomly with equal probability within the interval. The orbital separation of the circular inner binary was taken to be flat in $\log$ space between $1 R_{\odot}$ and $100 R_{\odot}$. We adopted these parameters from the population synthesis calculation of LMXBs (Willems \& Kolb 2003), in particular using the results of their models KM25 to KM100 of (Willems \& Kolb 2003).

The mass of the tertiary star was selected to be less massive than the secondary, but not smaller than $0.8 M_{\odot}$ (in theory there is no lower limit for the mass of the tertiary star). The outer orbital separation was chosen with a probability distribution flat in log space with a maximum of $10^{4} R_{\odot}$. The minimum separation was chosen to be consistent with a dynamically stable initial triple (adopting a $10 M_{\odot}$ primary and an inner orbital separation of $200 R_{\odot}$ ) and the earlier selected secondary and tertiary masses. The latter two stars were assumed not to accrete any material throughout the common envelope and supernova explosion. The eccentricity of the outer orbit before the supernova was selected at random from the thermal distribution between a circular orbit and a maximum which was chosen such that the triple is dynamical stable. The selection of the minimum orbital separation of the outer star and its eccentricity therewith becomes an iterative procedure. The other orbital elements are selected randomly, as we described in Section 4.2.

For each system we calculate the effect of the combined Blaauw-Boersma and intrinsic velocity kick on the inner and the outer orbits; the latter kick was assumed to be isotropic. In our simulations, we varied the mass loss in the supernova and the velocity distribution of the asymmetric kick. For electroncapture supernovae (Podsiadlowski et al. 2004) the fraction of surviving triples is one-third. For higher kick velocities, we adopted that the exploding star was $2.7 M_{\odot}$ and as a consequence the fraction of survivors drops to $1 / 25$ (Arzoumanian et al. 2002), $1 / 28$ (Hobbs et al. 2005), and $1 / 50$ (Cordes \& Chernoff 1998), where the quoted literature refers to the adopted kick velocity distribution. Note here that the smaller amount of mass lost in the electron-capture supernova explosions helps considerably in preserving more triples, as opposed to the more violent kicks.

We conclude that for every three inner binaries that survive the electron-capture supernova the outer tertiary star remains in orbit around the inner binary, but that this fraction may drop considerably (to $1 / 50$ ) when more mass is ejected in the supernova shell and the kick velocity is higher. Varying the amount of mass lost in the supernova explosion has a profound effect on the survivability of the triple, in particular since the Blaauw-Boersma kick imparted on the inner binary is proportional to this mass loss, and to the relative orbital velocity of the inner binary. It is interesting to note that the vast majority of the triples that survive the supernova explosion are dynamically stable, but their orbital eccentricities tend to be considerably higher than that indicated by the thermal distribution.

\subsection{The Fraction of Surviving Triples That Lead to Systems Like J1903+0327}

We synthesize the Galactic population of binaries like J1903+0327 by randomly selecting $10^{4}$ triples that survived the supernova explosion, and continue their evolution in AMUSE (see Section 4.1). Instead of performing a self-consistent evolution as adopted in Section 4.2 to validate the proposed scenario, we tentatively decoupled the mass-transfer process from the orbit integration. The population synthesis simulations start by resolving the mass transfer in the inner binary until the triple becomes dynamically unstable (Mardling \& Aarseth 2001), after which we continue the simulation by resolving the dynamics of the three-body system until one star is ejected. During this latter part we ignore the mass transfer.

Note that this decoupled approach, though computationally cheaper by about a factor of $10^{3}$, is not a priori less reliable than the self-consistent simulations in Section 4.2, because our numerical methods provide no self-consistent way to resolve the mass-transfer process in eccentric orbits.

The majority ( $\gtrsim 90 \%$ ) of our simulations lead to the disruption of the triple, which results in the ejection of the initial primary (MSP), the secondary (partially stripped sub-giant or white dwarf), or the tertiary (main-sequence F, G, or K dwarf) star, leaving the other two stars in a binary. The respective ratios at which these occur are $0.013,0.683$, and 0.304 for circular outer orbits (which is unlikely after the supernova) and 0.03, 0.489, and 0.481 for highly eccentric $(e \sim 0.9)$ orbits. For extremely high eccentricities $(e \sim 0.99)$, these fractions become 0.05, 0.75 , and 0.20 , respectively. The outer orbits tend to have high eccentricities because they often barely survive the supernova kick, and as a consequence, the total fraction of systems in which the MSP remains in a binary with the original outer component is $\lesssim 0.5$; the fraction of single MSPs is $\sim 0.05$.

The two methods through which we resolved the triples (self-consistent, see Section 4.1, as opposed the here adopted decoupled approach) give slightly different results, but these can be related to the variation of the implementations. The largest effect is illustrated in Figure 2, where we demonstrate how the slow changes in the inner orbit drive the secular evolution of the outer orbit. The main consequences of this coupling are the higher mass of the NS by the time the triple becomes dynamically unstable and the small fraction of triples that survive the entire evolution because the adiabatic expansion of the outer orbit prevents the triple from becoming dynamically unstable. The consequential loss of systems, however, is well compensated by the increased probability that the donor in the LMXB is ejected when the triple becomes dynamically unstable. The differences between the two numerical implementations affect our estimates for the birthrate (see Section 5.4) on a $\lesssim 20 \%$ level. For clarity, the statistical uncertainties between the two different numerical approaches depend on Poissonian arguments rather than on the lack of our understanding of parts of the physical process, such as the common-envelop evolution, the process of mass transfer in non-circular orbits, and the nonlinear effects in the dynamically unstable configuration just before the triple is resolved. We control the latter by adopting a high order and numerically extremely precise algorithm (see Section 4.1). However, the tipping-point physics of ejecting the MSP, its close white dwarf companion or the main-sequence outer star remains elusive, even when integrating near machine precision.

\subsection{The Number of J1903+0327-like Systems in the Galaxy}

The ratio of the birthrates of ordinary LMXBs to systems like $\mathrm{J} 1903+0327$ is a combination of the factors derived above: a few percent for the number of suitable triples, a reduction of a factor of $\sim 3$ owing to the effect of an electron-capture supernova, and then a fraction $\lesssim 0.5$ of systems in which the inner secondary is 
ejected. For larger average kick velocities (Cordes \& Chernoff 1998; Arzoumanian et al. 2002), the fraction of triples that survive the supernova drops from $1 / 3$ to $1 / 50$ (see Section 5.2). The higher kicks cause an even stronger reduction in the number of single MSPs, because outer orbits tend to be highly eccentric, which leads to a reduction of the probability that the MSP is ejected once the triple becomes dynamically unstable. The birthrate for systems like J1903+0327 is then in the range of $2 \times 10^{-4}$ to $3 \times 10^{-3}$ times the birthrate of ordinary LMXBs. Estimates of the total number of LMXBs in the Galaxy are in the range $10^{4}-10^{5}$ (Cote \& Pylyser 1989). The typical lifetime of an LMXB is of the order of a Gyr, whereas the lifetime of systems like J1903+0327 is limited by the lifetime of the MSP and the difference between that of the inner secondary star and the outer tertiary star. Both stars have a mass of the order of $1 M_{\odot}$ and their lifetimes exceed several Gyr, which is a factor of a few longer than the lifetime of the LMXB phase. The number of $\mathrm{J} 1903+0327$-like systems in the Galaxy then is at least 30-300 for electron-capture supernovae but drops to 3-30 when we adopt considerably more mass to be lost in the supernova and higher velocity kicks (Cordes \& Chernoff 1998; Arzoumanian et al. 2002).

\section{DISCUSSION}

We went through considerable effort to explain the existence of $\mathrm{J} 1903+0327$ as the result of the complex evolution in a hierarchical triple-star system. In this section, we will argue that our proposed model, as unlikely as it sounds, is currently the only viable model available, and that all other existing models fail to explain all the characteristics of J1903+0327. After that we will indicate a number of other sources that have evolved in quite a similar way, and that therefore support the triple scenario.

\subsection{Shortcomings of Earlier Models for J1903+0327}

In the triple scenario for the formation of $\mathrm{J} 1903+0327$ proposed by Champion et al. (2008), the observed $\sim 95$ day orbital period is that of an unobserved massive $0.9-1.1 M_{\odot}$ white dwarf, while the observed $\mathrm{G}$ dwarf has a considerably wider orbit. The observed eccentricity of the inner orbit would in this case be driven by the secular evolution via the Kozai mechanism (Kozai 1962). This scenario has a number of serious shortcomings, one of which is the unusually high white dwarf mass, which leaves little room to produce a massive $1.67 M_{\odot}$ NS. Since its discovery, the observed change in eccentricity, $\dot{e} \sim$ $10^{-16} \mathrm{~s}^{-1}$, is three orders of magnitude smaller than predictions based on the Kozai mechanism (Gopakumar et al. 2009), which excludes the presence of a highly inclined tertiary star. We exclude this scenario therefore from further consideration.

An alternative to the previous model would be the direct formation of a MSP in a supernova explosion, or via the fallback of material in a circum NS disk (Liu \& Li 2009). A variety of arguments against the direct formation of rapidly spinning pulsar with a small surface magnetic field $\left(2 \times 10^{8} \mathrm{G}\right)$ was provided by Champion et al. (2008). These include the following. First, of the $50 \mathrm{NSs}$ in young supernova remnants, none are fastspinning low-field pulsars (Kaspi \& Helfand 2002). Second, a "born-fast" scenario for J1903+0327 would likely account for the 15 isolated MSPs detected in the galactic disk, but the spin distribution, space velocities, and energetics of these single MSPs are indistinguishable from those of recycled, not "born fast" binary MSPs (Archibald et al. 2009), while their space velocities and scale heights do not match those of non- recycled single pulsars (Lorimer et al. 2007). Third, magnetic fields in young pulsars likely originate either from dynamo action in the proto-NS (Thompson \& Duncan 1993) or through compression of "frozen-in" fields of the progenitor star during collapse (Ferrario \& Wickramasinghe 2006), and no young pulsars with $B \lesssim 10^{10}$ Gauss, like J1903+0327, are known. We add a fourth argument: before the direct collapse, the NS progenitor ascends the giant branch star and common-envelope evolution with the 95 day-orbit G dwarf would have dramatically reduced the orbital period, in the same way as normal LMXBs form. For these four reasons, a core-collapse "born-fast" MSP can be ruled out.

The above arguments hold equally well against the accretioninduced collapse of a massive and rapidly rotating white dwarf to an NS. While this may lead to millisecond rotation periods, the NS formed here will be just as hot and differentially rotating during the early liquid phases as an NS formed by core collapse. In both cases, $10^{53}$ erg in gravitational energy is released, which will erase all memory of the violent mechanism in which the NS was formed. Therefore, dynamo action in both cases will not depend on the formation mechanism, and there is no fundamental reason for expecting a weak magnetic field in NSs formed by accretion-induced collapse. Furthermore, the direct accretion-induced collapse of a white dwarf to an MSP in population studies produces binaries with a orbital period $\lesssim 20$ days, which is considerably shorter than observed in $\mathrm{J} 1903+0327$ (Chen et al. 2011). In addition, this scenario requires the white dwarf to accrete from an evolved companion star, which is inconsistent with the observed main-sequence companion G star. This model thus fails to explain J1903+0327.

Finally, a formation scenario where J1903+0327 is formed when the donor star in the inner binary is ablated and destroyed, such as the case for the "black-widow" system PSR B1957+20 (Fruchter et al. 1988), has thee problems. First, the observed timescales for straightforward evaporation of the donor star are too long (Champion et al. 2008). Second, formation of such a system likely involves an exchange interaction (King et al. 2003), which would be greatly impeded by the outer G dwarf companion. Third, even if no exchange took place, the slow evaporation of the donor in the inner binary requires the triple to be dynamically stable with respect to the $G$ star in its current orbit. Since the supernova explosion can at most account for a reduction of a factor of two in the orbital separation, the common envelope should in that case be responsible for a further reduction from the initial orbital separation of $\gtrsim 560 R_{\odot}$ to the currently observed $\sim 123 R_{\odot}$, which requires considerable fine tuning. We therefore conclude that $\mathrm{J} 1903+0327$ is unlikely to have originated through a black-widow-like scenario.

Each of the above models has serious shortcomings, and we conclude that none of the scenarios discussed above give a satisfactory explanation for the formation of J1903+0327.

\subsection{The Missing Link}

There are currently no known triples with parameters suitable for evolving into systems like J1903+0327. However, there is observational evidence that such triples exist, as it is proposed that 4U 2129+47 (V1727 Cyg), a 5.24 hr LMXB, is accompanied by a spectral type $\mathrm{F}$ dwarf in an eccentric orbit of about 175 days (Garcia et al. 1989; Bothwell et al. 2008; Lin et al. 2009). This triple could have formed in the same way as J1903+0327, with the exception that after the common envelope and the subsequent supernova explosion, the inner binary period was smaller than the bifurcation period of about one day (Pylyser \& Savonije 
$1988,1989)$. The consequence of such a short orbital period is that the LMXB evolves to an even shorter orbital period. Interestingly the 175 day orbit of the outer star is too small to have been dynamically stable at the birth of the triple (Mardling \& Aarseth 2001). We argue that the common envelope and/or the supernova may have reduced the orbital separation of the outer star.

We validated the probability that a binary orbit shrinks as a result of the supernova by means of population synthesis and conclude that in a fraction of 0.511 of the triples that survive the supernova the separation of the circularized outer orbit is smaller than the initial orbit. However, if the progenitor of 4U 2129+47 had parameters comparable to what we derived for J1903+0327 in Section 2, the common-envelope phase of the inner binary must have resulted in a reduction of the outer orbit as well, by $\gtrsim 210 R_{\odot}$. Such a reduction in the separation of the outer orbit by the common envelope enormously boosts the survivability of the triple in the supernova explosion (see Figure 4), and therefore dramatically increases the birthrate of binaries like J1903+0327.

Following the same scenario as for forming 4U 2129+47 but with a less massive $\left(\lesssim 8 M_{\odot}\right)$ initial inner primary star, the inner binary could evolve into a cataclysmic variable such as EC 19314-5915, with an orbital period of $4.75 \mathrm{hr}$. The observed radial velocity of $\sim 9 \mathrm{~km} \mathrm{~s}^{-1}$ has been attributed to a G8 dwarf in orbit around the cataclysmic variable (Buckley et al. 1992), which is consistent with a semimajor axis of $\sim 2400 R_{\odot}$.

The real missing link would be the discovery of a relatively wide $\left(\gtrsim 30 R_{\odot}\right)$ LMXB that is orbited by a tertiary low-mass main-sequence star. The mass-transfer phase in our simulations averaged about $23 \mathrm{Myr}$ (see Section 4.2), while the lifetime of the MSP in J1903+0327 is at least 1 Gyr. We therefore expect that the Galaxy contains at most seven such wide triple LMXBs.

\subsection{The Effect of a Time-variable Mass-transfer Rate}

To study the effect of the adopted mass-transfer rate in the LMXB phase, we performed several simulations using the time-variable mass-transfer rates from detailed simulations of LMXBs (Tauris \& Savonije 1999). The consequence is that shortly after the onset of Roche lobe contact the rate of mass transfer exceeds our adopted constant rate of $\langle\dot{m}\rangle=$ $6 \times 10^{-10}\left(P_{\text {orb }}\right.$ (initial) $/ 1$ day) $M_{\odot} \mathrm{yr}^{-1}$ to drop below that rate at later time (see Section 4.1). This more realistic mass-transfer rate causes the inner orbits to expand more dramatically shortly after the start of the mass-transfer process and results on average in slightly lower NS masses and somewhat wider orbits. Though we did not further study the consequences of this more realistic approach, we realize that the more dramatic widening of the inner orbit in the early LMXB phase causes an increase of the birthrate of systems like J1903+0327.

\section{CONCLUSIONS}

We discussed the evolution of triple-star systems through a range of dramatic events, including several tidal circularizations, a common-envelope phase, a supernova and a stable phase of mass transfer that eventually leads to a dynamical instability in which one star is ejected.

In particular for producing a system like J1903+0327 we require a triple to be born as a rather ordinary dynamically stable hierarchical system of which the inner binary consists of a $9-13 M_{\odot}$ and a $0.8-2.0 M_{\odot}$ star in a $\gtrsim 200 R_{\odot}$ separation. This binary is orbited by a main-sequence star with a mass smaller than the initial secondary $\left(<2.0 M_{\odot}\right)$ with a semimajor axis $\gtrsim 560 R_{\odot}$. The chance that the triple survives the inevitable chain of events is not large but the result is profound and provides a satisfactory explanation for a number of known systems in the Galaxy, including J1903+0327, 4U 2129+47 (see Section 6.2), and EC 19314-5915.

The range in possible observable stages in the evolutionary sequence for forming a system like J1903+0327 sensitively depends on the orbital separation of the inner binary after the supernova, which provides the distinction between evolving into a binary MSP like J1903+0327 or an LMXB with an outer tertiary companion like $4 \mathrm{U} 2129+47$. The evolution of the triple naturally leads to a cataclysmic variable like EC 19314-4915, if in addition to a short post-common-envelope period the initial primary star evolves into a white dwarf rather than an NS. We expect that such triple CVs are rather common in the Galaxy.

We demonstrated that our model can indeed reproduce J1903+0327 qualitatively, and we estimate the number in the Galaxy by performing extensive population synthesis of postcommon-envelope triple systems (see Section 5). Our starting conditions are the progenitor of an LMXB which is accompanied by a third low-mass companion in a relatively wide orbit. Based on the observed statistics for such systems, their survival in the electron-capture supernova explosion of an $\mathrm{ONeMg}$ star and the consequences of the dynamical instability which results from the mass transfer in the inner binary, we conclude that the formation rate of $\mathrm{J} 1903+0327$-like systems is $\sim 3 \times 10^{-3}$ times that of LMXBs. With a lifetime at least as long as that of LMXBs, and an estimated total number of $10^{4}-10^{5}$ LMXBs in the Galaxy (Cote \& Pylyser 1989), we expect at least 30-300 systems like J1903+0327 in the Galaxy and an order of magnitude smaller number of single MSPs. The longer lifetime of the MSP binary compared to LMXBs results in an increase of this number of a factor of a few. In the most pessimistic scenario, when we adopt a higher velocity kick, this number drops to about 3-30 MSP binaries like J1903+0327 in the Galaxy, and a few single MSPs.

With a birthrate for Galactic LMXBs of $3.2 \times 10^{-6} \mathrm{yr}^{-1}$ (Kalogera \& Webbink 1998) to $7 \times 10^{-6} \mathrm{yr}^{-1}$ (Cote \& Pylyser 1989), we conclude that systems like J1903+0327 form at a rate of $\lesssim 2.1 \times 10^{-8}$, and a ten times smaller rate for single MSPs. These low rates makes the proposed scenario that the Galaxy should contain a few tens to hundreds of objects with characteristics similar to J1903+0327 unlikely, as we expected, but sufficiently probable, and consequently provides a satisfactory explanation for J1903+0327. Since the birthrate of single MSPs is expected to be quite similar to that of LMXBs (Dai \& Li 2010), our proposed triple scenario does not significantly contribute to the formation of single MSPs.

This work was supported by the Netherlands Research Council NWO (via grants 643.200.503, 639.073.803, and 614.061.608), the European Commission (grant FP7-PEOPLE2007-4-3-IRG 224838) and the Netherlands Research School for Astronomy (NOVA) for their support to AMUSE. Computing resources were provided by ASTRON. We thank Ron Taam for insightful discussions on common-envelope evolution at the MODEST-10 meeting in Beijing, and the AMUSE development team, Arjen van Elteren, Inti Pelupessi, Nathan de Vries and Marcel Marosvolgyi, for their much appreciated support, assistance, and feedback. We thank the editor of Nature, Leslie Sage, and for anonymous Nature referees for thoughtful 
comments on an earlier version of this paper submitted on 2010 September 15.

\section{REFERENCES}

Aarseth, S. J., \& Zare, K. 1974a, Celest. Mech., 10, 185

Aarseth, S. J., \& Zare, K. 1974b, Celest. Mech., 10, 516

Alpar, M. A., Cheng, A. F., Ruderman, M. A., \& Shaham, J. 1982, Nature, 300, 728

Archibald, A. M., et al. 2009, Science, 324, 1411

Arzoumanian, Z., Chernoff, D. F., \& Cordes, J. M. 2002, ApJ, 568, 289

Bhattacharya, D., \& van den Heuvel, E. P. J. 1991, Phys. Rep., 203,

Blaauw, A. 1961, Bull. Astron. Inst. Netherlands, 15, 265

Boersma, J. 1961, Bull. Astron. Inst. Netherlands, 15, 291

Bothwell, M. S., Torres, M. A. P., Garcia, M. R., \& Charles, P. A. 2008, A\&A, 485,773

Brandt, N., \& Podsiadlowski, P. 1995, MNRAS, 274, 461

Buckley, D. A. H., O’Donoghue, D., Kilkenny, D., Stobie, R. S., \& Remillard, R. A. 1992, MNRAS, 258, 285

Champion, D. J., et al. 2008, Science, 320, 1309

Chen, W., Liu, X., Xu, R., \& Li, X. 2011, MNRAS, 410, 1441

Cordes, J. M., \& Chernoff, D. F. 1998, ApJ, 505, 315

Cote, J., \& Pylyser, E. H. P. 1989, A\&A, 218, 131

Dai, H., \& Li, X. 2010, Sci. China G, 53, 125

Deloye, C. J., \& Taam, R. E. 2010, ApJ, 719, L28

Dewey, R. J., \& Cordes, J. M. 1987, ApJ, 321, 780

Dewi, J. D. M., Podsiadlowski, P., \& Pols, O. R. 2005, MNRAS, 363 , L71

Eggleton, P. 2006, Evolutionary Processes in Binary and Multiple Stars (Cambridge: Cambridge Univ. Press)

Eggleton, P. P., \& Kiseleva, L. G. 1996, in NATO ASIC Proc. 477, Evolutionary Processes in Binary Stars, ed. R. A. M. J. Wijers, M. B. Davies, \& C. A. Tout (Dordrecht: Kluwer), 345

Eggleton, P. P., \& Tokovinin, A. A. 2008, MNRAS, 389, 869

Eggleton, P. P., \& Tokovinin, A. A. 2010, VizieR Online Data Catalog, 738, 90869

Ferrario, L., \& Wickramasinghe, D. 2006, MNRAS, 367, 1323

Fregeau, J. M., Cheung, P., Portegies Zwart, S. F., \& Rasio, F. A. 2004, MNRAS, 352,1

Freire, P. C. C., et al. 2009, arXiv:0907.3219

Fruchter, A. S., Stinebring, D. R., \& Taylor, J. H. 1988, Nature, 333, 237

Garcia, M. R., Bailyn, C. D., Grindlay, J. E., \& Molnar, L. A. 1989, ApJ, 341, L75

Ge, H., Hjellming, M. S., Webbink, R. F., Chen, X., \& Han, Z. 2010, ApJ, 717, 724
Gopakumar, A., Bagchi, M., \& Ray, A. 2009, MNRAS, 399, L123

Hansen, B. M. S., \& Phinney, E. S. 1997, MNRAS, 291, 569

Heggie, D. C. 1975, MNRAS, 173, 729

Hills, J. G. 1983, ApJ, 267, 322

Hobbs, G., Lorimer, D. R., Lyne, A. G., \& Kramer, M. 2005, MNRAS, 360, 974 Kalogera, V., \& Webbink, R. F. 1998, ApJ, 493, 351

Kaspi, V. M., \& Helfand, D. J. 2002, in ASP Conf. Ser. 271, Neutron Stars in Supernova Remnants, ed. P. O. Slane \& B. M. Gaensler (San Francisco, CA: ASP), 3

King, A. R., Davies, M. B., \& Beer, M. E. 2003, MNRAS, 345, 678

Kozai, Y. 1962, AJ, 67, 591

Kozai, Y. 1979, in IAU Symp. 81, Dynamics of the Solar System, ed. R. L. Duncombe (Cambridge: Cambridge Univ. Press), 231

Lajoie, C., \& Sills, A. 2011a, ApJ, 726, 66

Lajoie, C., \& Sills, A. 2011b, ApJ, 726, 67

Lin, J., Nowak, M. A., \& Chakrabarty, D. 2009, ApJ, 706, 1069

Liu, X., \& Li, X. 2009, ApJ, 692, 723

Lorimer, D. 2008, Living Rev. Relativ., 11, 8

Lorimer, D. R., McLaughlin, M. A., Champion, D. J., \& Stairs, I. H. 2007, MNRAS, 379, 282

Lyne, A. G., \& Lorimer, D. R. 1994, Nature, 369, 127

Mardling, R. A., \& Aarseth, S. J. 2001, MNRAS, 321, 398

Podsiadlowski, P., Langer, N., Poelarends, A. J. T., Rappaport, S., Heger, A., \& Pfahl, E. 2004, ApJ, 612, 1044

Portegies Zwart, S., et al. 2009, New Astron., 14, 369

Portegies Zwart, S. F. 1995, A\&A, 296, 691

Pylyser, E., \& Savonije, G. J. 1988, A\&A, 191, 57

Pylyser, E. H. P., \& Savonije, G. J. 1989, A\&A, 208, 52

Radhakrishnan, V., \& Srinivasan, G. 1982, Curr. Sci., 51, 1096

Scheck, L., Plewa, T., Janka, H., Kifonidis, K., \& Müller, E. 2004, Phys. Rev. Lett., 92, 011103

Schwab, J., Podsiadlowski, P., \& Rappaport, S. 2010, ApJ, 719, 722

Shore, S. N., Livio, M., \& van den Heuvel, E. P. J. 1994, in Saas-Fee Advanced Course 22, Interacting Binaries, ed. H. Nussbaumer \& A. Orr (Berlin: Springer), 210

Taam, R. E. 1983, ApJ, 270, 694

Taam, R. E., \& Sandquist, E. L. 2000, ARA\&A, 38, 113

Tauris, T. M., \& Savonije, G. J. 1999, A\&A, 350, 928

Tauris, T. M., \& Takens, R. J. 1998, A\&A, 330, 1047

Thompson, C., \& Duncan, R. C. 1993, ApJ, 408, 194

van den Heuvel, E. P. J. 2004, in 5th INTEGRAL Workshop on the INTEGRAL Universe, ed. V. Schoenfelder, G. Lichti, \& C. Winkler (ESA SP-552; Noordwijk: ESA), 185

Webbink, R. F. 1984, ApJ, 277, 355

Webbink, R. F., Rappaport, S., \& Savonije, G. J. 1983, ApJ, 270, 678

Willems, B., \& Kolb, U. 2003, MNRAS, 343, 949 